\title{
Effect of bulk modulus on performance of a hydrostatic transmission control system
}

\author{
ALI VOLKAN AKKAYA \\ Yildiz Technical University, Mechanical Engineering Department, 34349, \\ Besiktas, Istanbul, Turkey \\ e-mail: aakkaya@yildiz.edu.tr
}

MS received 9 September 2005; revised 20 February 2006

\begin{abstract}
In this paper, we examine the performance of PID (proportional integral derivative) and fuzzy controllers on the angular velocity of a hydrostatic transmission system by means of Matlab-Simulink. A very novel aspect is that it includes the analysis of the effect of bulk modulus on system control. Simulation results demonstrates that bulk modulus should be considered as a variable parameter to obtain a more realistic model. Additionally, a PID controller is insufficient in presence of variable bulk modulus, whereas a fuzzy controller provides robust angular velocity control.
\end{abstract}

Keywords. Hydrostatic transmission; bulk modulus; PID (proportional integral derivative); fuzzy controller.

\section{Introduction}

Hydrostatic transmission (HST) systems are widely recognized as an excellent means of power transmission when variable output velocity is required in engineering applications, especially in field of manufacturing, automation and heavy duty vehicles. They offer fast response, maintain precise velocity under varying loads and allow improved energy efficiency and power variability (Dasgupta 2000; Kugi et al 2000). A basic hydrostatic transmission is an entire hydraulic system. Generally, it contains a variable-displacement pump driven by an induction motor, a fixed or variable displacement motor, and all required controls in one simple package. By regulating the displacement of the pump and/or motor, a continuously variable velocity can be achieved (Wu et al 2004).

Manufacturers and researchers continue to improve the performance and reduce the cost of hydrostatic systems. Especially, modelling and control studies of hydrostatic transmission systems have attracted considerable attention in recent decades. Some studies on this topic can be found in the literature (Huhtala 1996; Manring \& Luecke 1998; Dasgupta 2000; Kugi et al 2000; Dasgupta et al 2005). Various rotational velocity control algorithms for hydrostatic systems are developed and applied by Lennevi \& Palmberg (1995), Lee \& Wu (1996), Piotrowska (2003). All these designs use the bulk modulus as a fixed value through a wide pressure range. However, in practice, the bulk modulus is an essential part of dynamic behaviours of 
the hydraulic systems (McCloy \& Martin 1980; Watton 1989). Due to temperature variations and air entrapment, the bulk modulus may vary during the operation of the hydraulic systems (Eryilmaz \& Wilson 2001). A little entrapped air is enough to reduce the bulk modulus significantly (Merrit 1967; Tan \& Sepehri 2002). Moreover, system pressure plays an important role on the bulk modulus value (Wu et al 2004). Some effects of instabilities induced by bulk modulus nonlinearities such as pressure oscillations in the form of pressure waves can be detrimental to operation of hydraulic systems and may result in reduced component life, loss of performance, disturbance in control systems, reduced efficiency and increased acoustic noise. In spite of these adverse effects, there are few studies about bulk modulus within hydrostatic transmission systems. Yu et al (1994) developed an on-line parameter identification method, determining the effective oil bulk modulus within an actual hydraulic system by measuring the propagation of a pressure wave through a long pipe. Marning (1997) developed a linear relation between oil bulk modulus and pressure for a HST system. However, to date, nothing has appeared in the literature that addresses the effect of bulk modulus dynamics incorporated into a hydrostatic transmission model on control design process of the HST system. In fact, models of hydrostatic transmission systems with variable bulk modulus have more complex dynamic behaviour than normal. Moreover, having servo control of the system, dynamics of bulk modulus becomes more important because the closed-loop system itself raises the issue of stability.

Bulk modulus cannot be determined directly and hence needs to be estimated. Based on this estimation, corrective actions may be taken in control applications for HST systems. The complex dynamic interactions between variable bulk modulus and the control action is investigated using modelling and simulation analysis. Simulation tests are particularly beneficial when preparing a model of a real system is complicated and time-consuming. A servo hydrostatic transmission control system is a good example for this issue. The determination of static and dynamic behaviours using simulation tests is possible without expensive prototypes. The simulation also makes a shorter product-designing cycle possible.

This study focuses on control performance of a typical HST system. A nonlinear model of the system is studied by means of Matlab-Simulink software. The system model is a combination of each individual component model consisting of pump, valve, hydraulic hose and hydraulic motor. In addition, the variable bulk modulus is presented to describe the effects of this phenomenon on system dynamics and control algorithm. For this purpose, two different hydraulic hose Simulink models are incorporated separately into the system model. In addition, the models are utilized in the control design process. The control of the angular velocity of the hydraulic motor coupled with load is achieved by PID (proportional integral derivative) and fuzzy types of controller. In the first model, bulk modulus is assumed to have a fixed value and angular velocity control of the HST system is carried out with the classical PID control algorithm. In the second model, bulk modulus is defined as a variable parameter depending on entrapped air and system pressure. This new model is applied on velocity control of the HST system under the same PID control parameters. In the following, fuzzy controller is implemented in this new model in order to judge its capability against variable bulk modulus nonlinearity. The simulation results of two control approaches are then compared to analyse the differences in the performance of the HST system in terms of bulk modulus dynamics.

\section{Mathematical model}

The physical model of the HST system considered for this study is shown in figure 1 . The variable displacement pump driven by an induction motor supplies hydraulic power to a fixed 


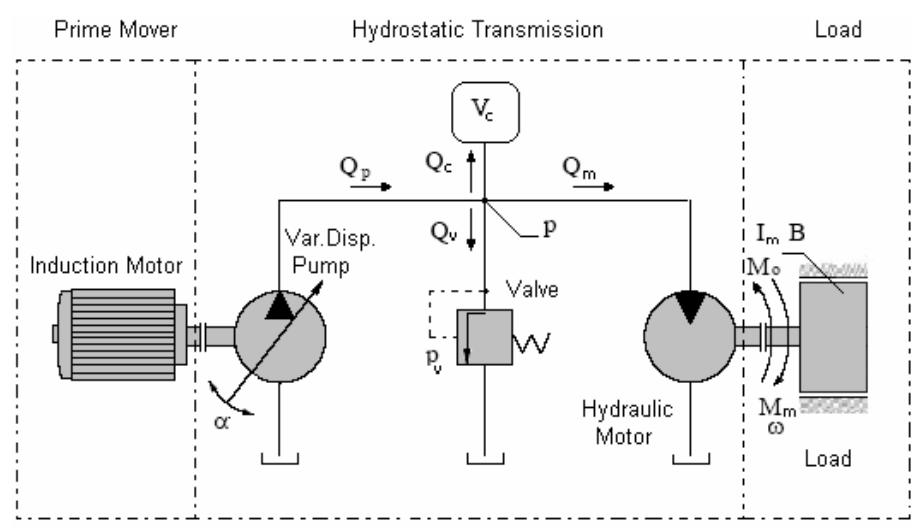

Figure 1. Hydrostatic transmission system.

displacement hydraulic motor for driving load. To protect the system from excessive pressure, a pressure relief valve is used.

From a research objective point of view, the descriptions of a system mathematical model should be as simple as possible. At the same time, it must include important characteristics of the real event. One way to understand the system is to separate the system into components for the purpose of modelling. Using a fundamental knowledge of physics, for instance the moment equilibrium and continuity equation, a model that represents the dynamics behaviour of each component can be derived at the component levels. Having understood each individual component, we can understand the overall system by interconnecting the components together to obtain an overall system model (Prasetiawan 2001). In this paper, the model of each component used for the HST system is developed using earlier methods (Jedrzykiewicz et al 1997, 1998).

\subsection{Variable-displacement pump}

It is assumed that the angular velocity of the prime mover (induction motor) is constant. Therefore, angular velocity of the pump shaft is constant. Pump flow rate can be adjusted with variable displacement via the swashplate displacement angle and can be given as

$$
Q_{p}=\alpha k_{p} \eta_{v p}
$$

where, $Q_{p}$ is pump flow rate $\left(\mathrm{m}^{3} / \mathrm{s}\right), \alpha$ is displacement angle of swashplate $\left(^{\circ}\right), k_{p}$ is pump coefficient $\left(\mathrm{m}^{3} / \mathrm{s}\right), \eta_{v p}$ is pump volumetric efficiency (-) which is assumed not to depend on pump rotation angle.

\subsection{Pressure relief valve}

To simplify, pressure relief valve dynamics is not taken into consideration. Therefore, two equation as below are given for passing flow rate through pressure relief valve $\left(\mathrm{m}^{3} / \mathrm{s}\right)$ in the state of opening and closing.

$$
\begin{aligned}
& Q_{v}=k_{v}\left(P-P_{v}\right), \text { if } P>P_{v}, \\
& Q_{v}=0, \text { if } P \leq P_{v},
\end{aligned}
$$


where, $k_{v}$ is slope coefficient of valve static characteristic $\left(\mathrm{m}^{5} / \mathrm{Ns}\right), P$ is system pressure ( $\left.\mathrm{Pa}\right)$ and $P_{v}$ is valve opening pressure $(\mathrm{Pa})$.

\subsection{Hydraulic hose}

As in traditional modelling, the pressurized hose that connects the pump to the motors is modelled as volume with a fixed bulk modulus in this section. Variable bulk modulus are discussed in the following subsection.

The fluid compressibility relation can be given as in (4). Equation (5) provides the pressure value from a given flow rate. It is assumed that pressure drop in the hydraulic hose is negligible.

$$
\begin{aligned}
Q_{c} & =(V / \beta)(\mathrm{d} P / \mathrm{d} t), \\
(\mathrm{d} P / \mathrm{d} t) & =(\beta / V) Q_{c},
\end{aligned}
$$

where, $Q_{c}$ is flow rate deal with fluid compressibility $\left(\mathrm{m}^{3} / \mathrm{s}\right), V$ is the fluid volume $\left(\mathrm{m}^{3}\right)$ subjected to pressure effect, $\beta$ is fixed bulk modulus $(\mathrm{Pa})$.

2.3a Variable bulk modulus Fluid is an important element of hydrostatic systems and enables power transmission, hence it can influence the dynamic behaviours of the system and the control system. The bulk modulus of non-aerated hydraulic oil depends on temperature and pressure, for mineral oils with additives its value ranges from 1200 to $2000 \mathrm{MPa}$. Moreover, system pressure and entrapped air affect the bulk modulus value. If a hydraulic hose is used rather than a steel pipe, the bulk modulus of this section may be considerably reduced. Owing to these reasons, the parameters influencing bulk modulus value must be included in the HST model for more accurate system dynamics.

The equation which gives the variable bulk modulus of fluid-air mixture in a flexible container is as follows (McCloy \& Martin 1980):

$$
\frac{1}{\beta_{v}}=\frac{1}{\beta_{\mathrm{f}}}+\frac{1}{\beta_{\mathrm{h}}}+\frac{V_{\mathrm{a}}}{V_{t}} \cdot \frac{1}{\beta_{\mathrm{a}}},
$$

where, the subcripts $\mathrm{a}, \mathrm{f}$ and $\mathrm{h}$ refer to air, fluid, and hose respectively. It is assumed that the initial total volume $V_{t}=V_{\mathrm{f}}+V_{\mathrm{a}}$, and that $\beta_{\mathrm{f}} \gg \beta_{\mathrm{a}}$. Thus bulk modulus will be less than any $\beta_{\mathrm{f}}, \beta_{\mathrm{h}}$, or $V_{t} / V_{\mathrm{a}} \beta_{\mathrm{a}}$. The bulk modulus of the fluid $\beta_{f}$ is obtained from the manufacturers data. The adiabatic bulk modulus used for air is $\left(C_{p} / C_{v}\right) P=1.4 P$. With these assumptions, (6) can be rewritten as in,

$$
\frac{1}{\beta_{v}}=\frac{1}{\beta_{\mathrm{f}}}+\frac{1}{\beta_{\mathrm{h}}}+\frac{s}{1 \cdot 4 \cdot P},
$$

where, $s$ is entrapped air percent in the total volume $\left(s=V_{\mathrm{a}} / V_{t}\right)$.

\subsection{Hydraulic motor and load}

Flow rate used in the hydraulic motor $\left(\mathrm{m}^{3} / \mathrm{s}\right)$ can be written as in

$$
Q_{m}=k_{m} \omega / \eta_{v m},
$$

where, $k_{m}$ is hydraulic motor coefficient $\left(\mathrm{m}^{3}\right), \omega$ is angular velocity of hydraulic motor $(1 / \mathrm{s})$ and $\eta_{v m}$ is volumetric efficiency of the motor (-). It is assumed that hydraulic motor efficiency does not depend on its shaft rotation angle. Hydraulic motor torque $(\mathrm{Nm})$ can be written as,

$$
M_{m}=k_{m t} \Delta P \eta_{m m},
$$


where, $k_{m t}$ is motor torque coefficient $\left(\mathrm{m}^{3}\right), \Delta P$ is pressure drop in hydraulic motor ( $\left.\mathrm{Pa}\right)$ and $\eta_{m m}$ is mechanical efficiency of hydraulic the motor (-). The torque produced in the hydraulic motor $(\mathrm{Nm})$ is equal to the sum of the moments from the motor loads and can be given as,

$$
M_{m}=M_{I}+M_{B}+M_{o},
$$

where, $M_{I}, M_{B}$ and $M_{o}$ are the moments resulting from load inertia, friction force and machine operation respectively. These moments can be denoted as

$$
M_{m}=I_{m}(\mathrm{~d} \omega / \mathrm{d} t)+B \omega+M_{o},
$$

where, $I_{m}$ is the inertia of the hydraulic motor shaft $\left(\mathrm{Nms}^{2}\right), B$ is viscous friction coefficient of motor and its shaft $(\mathrm{Ns} / \mathrm{m})$, and $\omega$ is angular velocity of motor shaft (1/s). Equation (11) can be used to determine the angular velocity of the hydraulic motor shaft. This equation is rearranged for angular velocity as

$$
\mathrm{d} \omega / \mathrm{d} t=\left(M_{m}-B \omega-M_{o}\right) / I_{m} .
$$

\subsection{Hydrostatic transmission system}

The fundamental mathematical models of the system components and phenomena occurring in hydrostatic systems are conveniently combined to obtain the overall HST system model. Accordingly, a hydrostatic transmission is modelled as a lumped system. In the development of the dynamic model of the system, it is assumed that static and dynamic features of the transmission do not depend upon the direction of hydraulic motor rotation and the transmission is a state of thermal balance. Leakage flows in pump and motor are not taken into account during the modelling.

The mathematical model of the HST system consists of two equations as below: equality of flow rate:

$$
Q_{p}=Q_{m}+Q_{c}+Q_{v},
$$

moment:

$$
M_{m}=M_{I}+M_{B}+M_{o} .
$$

Using (5) and (12), the following are then obtained,

$$
\begin{aligned}
\mathrm{d} P / \mathrm{d} t & =(\beta / V)\left(Q_{p}-Q_{m}-Q_{v}\right), \\
\mathrm{d} \omega / \mathrm{d} t & =\left(M_{m}-B \omega-M_{o}\right) / I_{m} .
\end{aligned}
$$

A commonly available general purpose simulation package Matlab/Simulink is used to solve the nonlinear equations. The Simulink model based on the component mathematical models of HST system is given in figure 2 . The component models can be easily modified in accordance width specific constructions. Accordingly, when bulk modulus is rebuilt in the hydraulic hose component with regard to (7), the second model can be generated. 


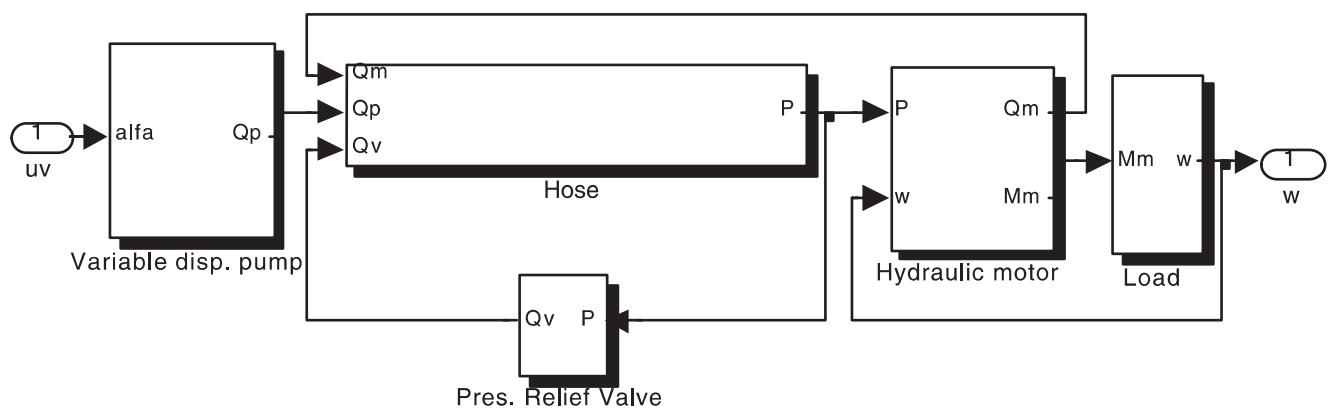

Figure 2. Simulink model of hydrostatic transmission system.

\section{Control applications}

Most publications related to the HST control are related to the speed control of the hydraulic motor connected to the load. In order to achieve this goal, different closed-loop control design strategies can be used. However, Lee \& Wu (1996) showed that using only pump displacement to regulate load speed is the most effective of all the methods they tested. In addition, Re et al (1996) concluded that the sole use of pump displacement actuation to control one load speed of a system with variable-displacement pump and motor is the most efficient, and should be always preferred whenever possible. For this reason, in the HST systems being considered in this study, the output angular velocity is controlled by the flow rate supplied to the hydraulic motor, and this flowrate is adjusted by the swashplate angle of the variable-displacement pump. Swashplate dynamics are not taken into consideration in the control application in this study for the sake of simplicity. In addition, the swashplate control system usually has faster dynamics than the rest of the system, and therefore neglecting its dynamics is justified (Watton 1989).

To precisely control the angular velocity of the hydraulic motor in hydrostatic transmission control systems, an appropriate controller must be designed in advance. In industrial applications, classical control methods such as PI, PID are being used for velocity control of HST systems. It is crucial to determine controller parameters accurately because PID control methods have linear characteristics. They are sometimes insufficient to overcome nonlinearities which exist in the nature of the HST systems for high precision applications (Tikkanen et al 1995; Prasetiawan 2001). In particular, the bulk modulus ought to be regarded as a source of significant nonlinearity for this type of controller. Thus, the controller has to be very robust to account for such wide variation. Use of knowledge-based systems in process control is increasing, especially in the fields of fuzzy control (Tanaka 1996). Unlike classical control methods, the fuzzy controller is designed with linguistic terms to cope with the nonlinearities. Therefore, this control method is also applied to judge its capacity to reduce the adverse effect of variable bulk modulus.

\subsection{PID control}

The structure of the PID control algorithm used for the angular velocity control of HST system is given in (17) and (18) below. Ziegler-Nichols method is implemented for tuning control parameters, such as proportional gain $\left(K_{p}\right)$, derivative time constant $\left(\tau_{d}\right)$ and integral time constant $\left(\tau_{i}\right)$ (Ogata 1990). After fine adjustments, the optimal control parameters are 


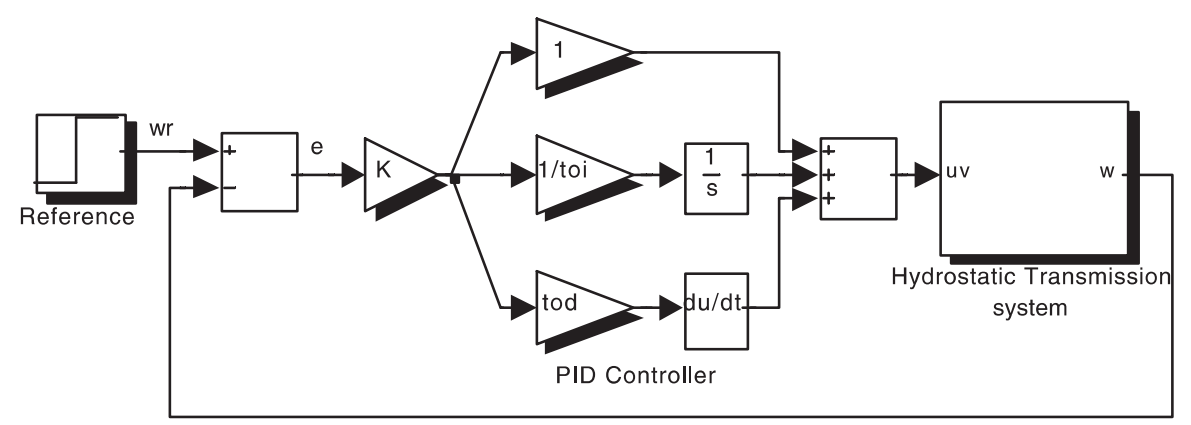

Figure 3. Simulink model of HST system for PID control.

determined for the reference angular velocity. Figure 3 shows the Simulink model of the PID-controlled HST system.

$$
\begin{aligned}
u v(t) & =K_{p} \cdot e(t)+K_{p} \cdot \tau_{d} \cdot \frac{\mathrm{d} e(t)}{\mathrm{d} t}+\frac{K_{p}}{\tau_{i}} \int e(t) \cdot \mathrm{d} t, \\
e(t) & =\omega_{r}-\omega .
\end{aligned}
$$

\subsection{Fuzzy control}

Fuzzy logic has come a long way since it was first presented to technical society, when Zadeh (1965) first published his seminal work. Since then, the subject has been the focus of many independent research investigations. The attention currently being paid to fuzzy logic is most likely the result of present popular consumer products employing fuzzy logic. The advantages of this method are its applicability to nonlinear systems, simplicity, good performance and robust character. These days, this method is being applied to engineering control systems such as robot control, flight control, motor control and power systems successfully.

In fuzzy control, linguistic descriptions of human expertise in controlling a process are represented as fuzzy rules or relations. This knowledge base is used by an inference mechanism, in conjunction with some knowledge of the states of the process in order to determine control actions. Unlike the conventional controller, there are three procedures involved in the implementation of a fuzzy controller: fuzzification of inputs, and fuzzy inference based on the knowledge and the defuzzification of the rule-based control signal. The structure of the fuzzy controller is seen in figure 4.

An applied fuzzy controller needs two input signals. These signals are error $(e)$ and derivative of error $(\mathrm{d} e)$ respectively. The usual overlapped triangular fuzzy membership functions are used for two input signals $(e, \mathrm{~d} e / \mathrm{d} t)$ and the output signal $(u)$. Figure 5 shows the structure of the membership functions of input and output signals. Input signals are transformed at intervals of $[-1,1]$ by scaling factors which are $\mathrm{G} e$ and Gde.

In the fuzzification process, all input signals are expressed as linguistic values which are: NB - negative big, NM - negative medium, NS-negative small, ZE-zero, PS-positive small, PM-positive medium, PB-positive big. After input signals are converted to fuzzy linguistic variables, these variables are sent to the inference mechanism to create output signals. 


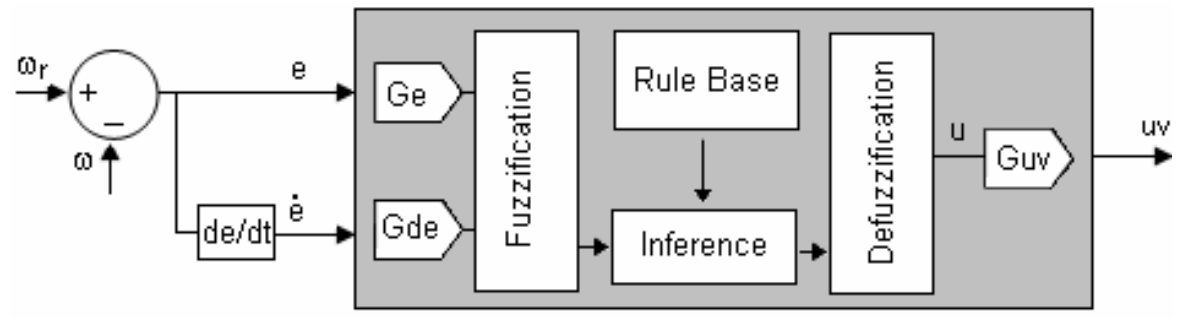

Figure 4. Structure of a fuzzy controller.

The inference process consists of forty nine rules driven by the linguistic values of the input signals. These fuzzy rules written as a rule base are shown given in table 1 . The rule base is developed by heuristics with error in motor angular velocity and derivation of error in this velocity. For instance, one of the possible rules is: IF $e=$ PS and d $e=$ NB THAN $u=$ NM. This rule can be explained as in the following: If the error is small, angular velocity of hydraulic motor is around the reference velocity. Significantly big negative value of derivation of error shows that the motor velocity is rapidly approaching the reference position. Consequently, controller output should be negative middle to prevent overshoot and to create a brake effect. As a rule-inference method, the Mamdani Method is selected because of its general acceptance (Tanaka 1996).

Defuzzification transforms the control linguistic variables into the exact control output. In defuzzification, the method of centre of gravity is implemented (Tanaka 1996), as

$$
u=\sum_{i=1}^{n} W_{i} B_{i} / \sum_{i=1}^{n} W_{i}
$$
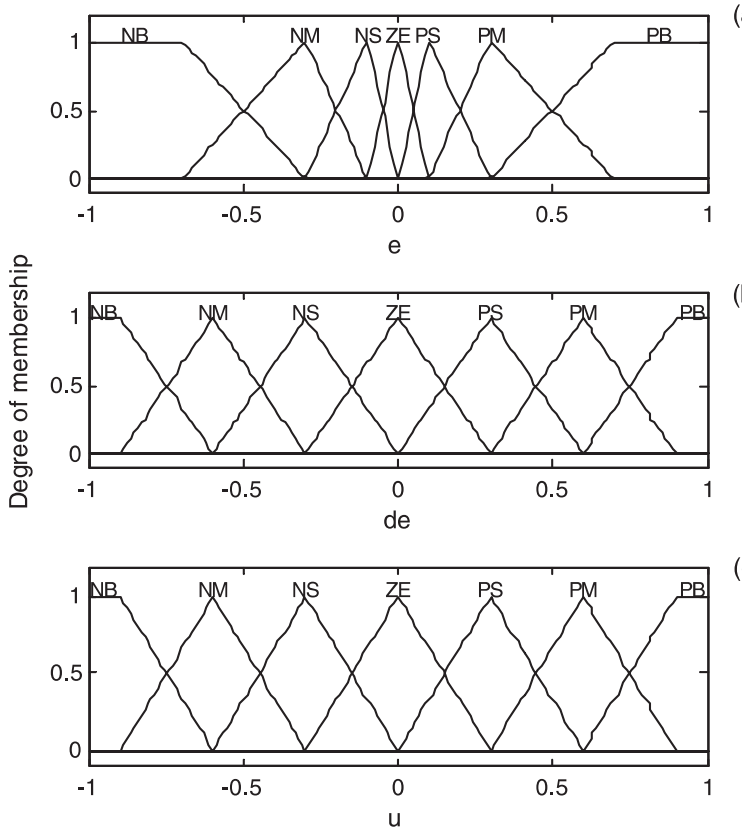

(a)

(b)

(c)
Figure 5. Triangular fuzzy membership functions, (a) $e$ input signal, (b) $\mathrm{d} e$ input signal, (c) $u$ output signals. 
Table 1. Rule base for fuzzy control.

\begin{tabular}{llllllll}
\hline $\mathrm{d} e \backslash e$ & $\mathrm{NB}$ & $\mathrm{NM}$ & $\mathrm{NS}$ & $\mathrm{ZE}$ & $\mathrm{PS}$ & $\mathrm{PM}$ & $\mathrm{PB}$ \\
\hline $\mathrm{NB}$ & $\mathrm{NB}$ & $\mathrm{NB}$ & $\mathrm{NB}$ & $\mathrm{NM}$ & $\mathrm{NM}$ & $\mathrm{NS}$ & ZE \\
$\mathrm{NM}$ & $\mathrm{NB}$ & $\mathrm{NB}$ & $\mathrm{NM}$ & $\mathrm{NS}$ & $\mathrm{NS}$ & ZE & PS \\
NS & NB & NM & NS & NS & ZE & PS & PM \\
ZE & NM & NS & NS & ZE & PS & PS & PM \\
PS & NM & NS & ZE & PS & PS & PM & PB \\
PM & NS & ZE & PS & PS & PM & PB & PB \\
PB & ZE & PS & PM & PM & PB & PB & PB \\
\hline
\end{tabular}

where, $u$ is the output signal of the fuzzy controller, $W_{i}$ is the degree of the firing of the $i$ th rule, $B_{i}$ is the centroid of the consequent fuzzy subset of $i$ th rule. Real values of control output signal $(u v)$ are determined by the scaling factor of Guv. As a result, the fuzzy controller built-in Fuzzy Logic Toolbox of the Matlab program has been added to the Simulink model of hydrostatic transmission system for simulation analysis (figure 6).

\section{Simulation results and discussion}

The validity of the influence of bulk modulus dynamics on HST control system has been tested in computer simulations. In order to carry out simulation, some physical and simulation parameters corresponding to HST system are taken from work of McCloy \& Martin (1980) and Jedrzykiewicz et al (1997, 1998), and other control parameters are as given in table 2.

Open loop pressure and angular velocity responses of the HST system are given in figures 7a and $\mathrm{b}$ respectively, under fixed bulk modulus and variable bulk modulus. Comparing the simulation results shows that the model including variable bulk modulus shows flexible dynamics and decreasing system stiffness (figure 7a). Moreover, a degree of aeration less than $1 \%$ brings about considerable changes of velocity and pressure responses because the aeration of the working fluid results in decrease of fluid bulk modulus and changes its characteristics as a function of pressure.

The dynamic behaviour patterns in figure 8 are obtained from PID control. It is observed from figure $8 \mathrm{a}$ that the system model including the fixed bulk modulus is in good agreement with reference velocity. In contrast to this, the simulation result of the model with variable bulk modulus show oscillations in the transient regime. The reason is that the bulk modulus

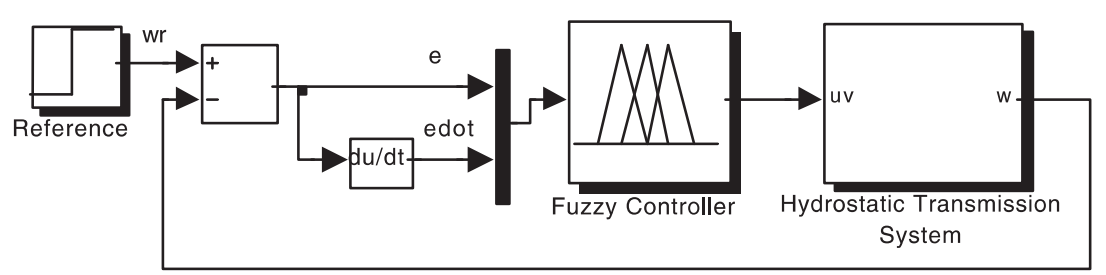

Figure 6. Simulink model of HST system for fuzzy controller. 
Table 2. Physical and simulation parameters.

Moments resulting from machine operation

Viscous friction coefficient

Displacement angle of swashplate

Inertia of hydraulic motor shaft

Opening pressure value of valve

Fluid volume subjected to pressure effect

Pump coefficient

Hydraulic motor coefficient

Motor torque coefficient

Slope coefficient of relief valve

Pump volumetric efficiency

Mechanical efficiency of hydraulic motor

Bulk modulus of fluid

Bulk modulus of hose

Degree of entrapped air

Reference angular velocity

Proportional gain constant

Derivative time constant

Integral time constant

Scaling factor of error

Scaling factor of derivative of error

Scaling factor of control signal

$\begin{array}{ll}M_{o}(\mathrm{Nm}) & 150 \\ B(\mathrm{Ns} / \mathrm{m}) & 15 \\ a\left({ }^{\circ}\right) & 16 \text { for open-loop } \\ I_{m}\left(\mathrm{Nms}^{2}\right) & 0.04 \\ P_{v}(\mathrm{~Pa}) & 12 \times 10^{6} \\ V\left(\mathrm{~m}^{3}\right) & 1.4145 \times 10^{-4} \\ k_{p}\left(\mathrm{~m}^{3} /{ }^{\circ} \mathrm{s}\right) & 2.688 \times 10^{-5} \\ k_{m}\left(\mathrm{~m}^{3}\right) & 3.979 \times 10^{-5} \\ k_{m t}\left(\mathrm{~m}^{3}\right) & 3.979 \times 10^{-5} \\ k_{v}\left(\mathrm{~m}^{5} / \mathrm{Ns}\right) & 0.2 \times 10^{-9} \\ \eta_{v p}(\%) & 97 \\ \eta_{m m}(\%) & 95 \\ \beta_{f}(\mathrm{~Pa}) & 1.49 \times 10^{9} \\ \beta_{h}(\mathrm{~Pa}) & 47 \times 10^{7} \\ s(\%) & 0.5 \\ \omega_{r}(1 / \mathrm{s}) & 7 \\ K_{p}(-) & 70 \\ \tau_{d}(-) & 0.0002875 \\ \tau_{i}(-) & 0.00115 \\ \mathrm{G} e(-) & 0.1 \\ \mathrm{Gd} e(-) & 0 \cdot 0001 \\ \mathrm{G} u(-) & 1500 \\ \end{array}$
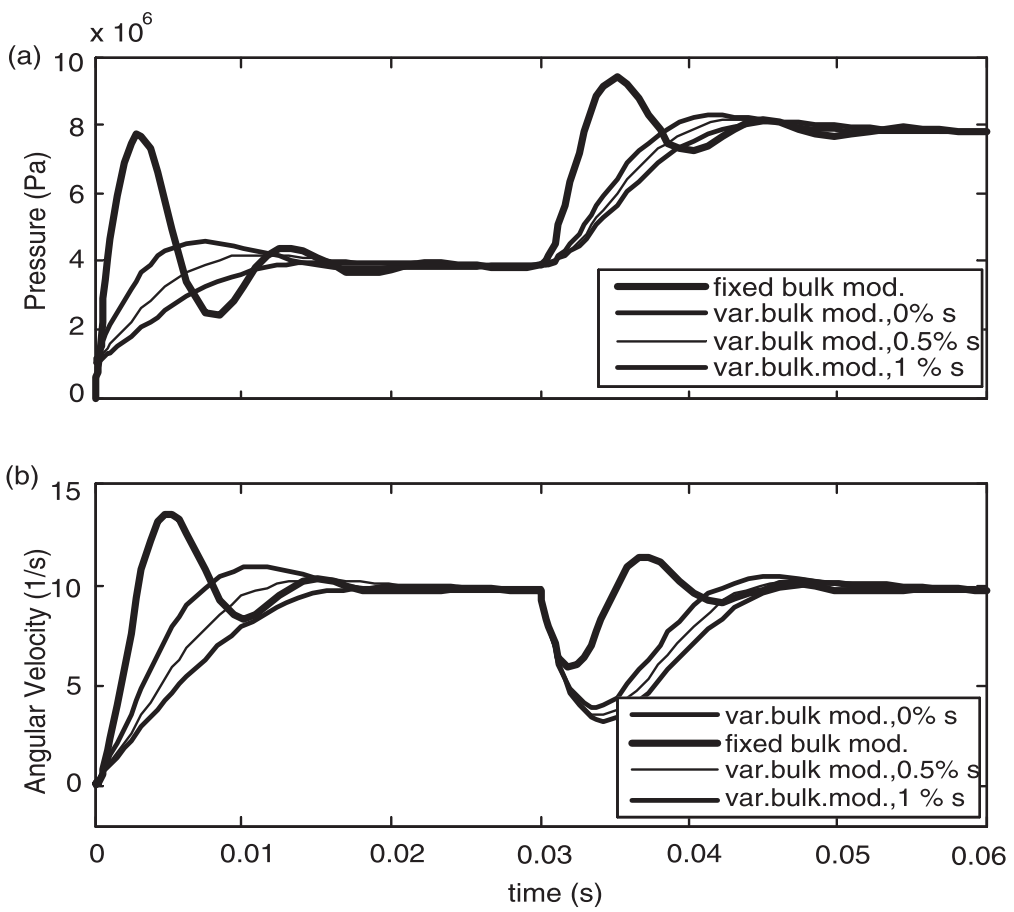

Figure 7. Open loop responses of HST system under fixed and variable (a) system pressure, (b) angular velocity. 
(a)

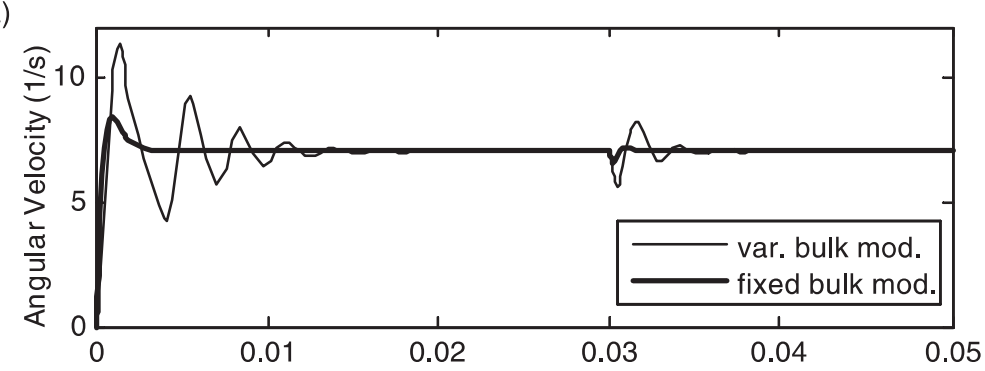

(b)

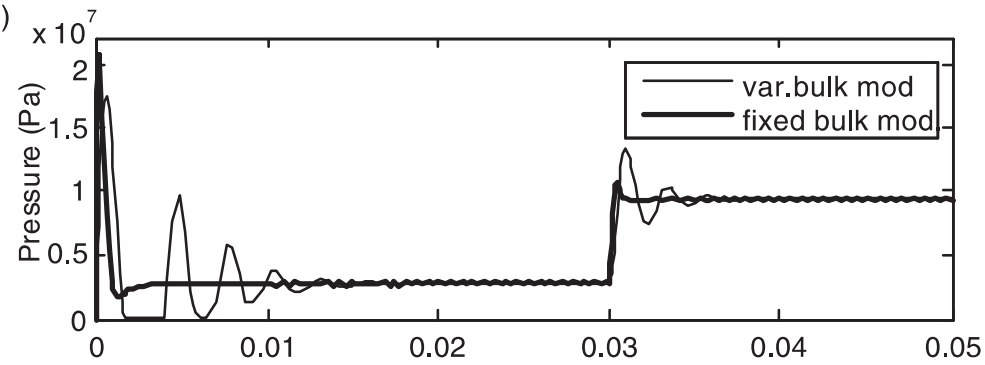

(c)

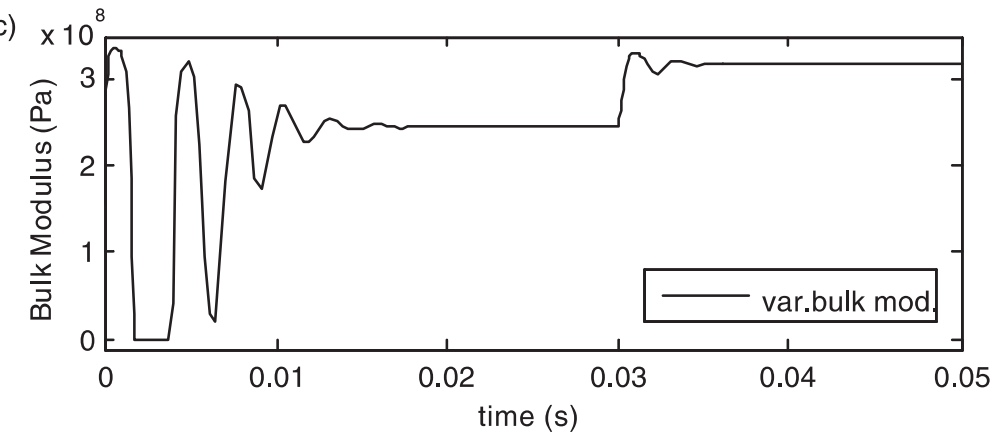

Figure 8. PID control responses of HST system for fixed and variable bulk modulus (a) angular velocity, (b) system pressure, (c) variable bulk modulus.

value becomes lower than that of the fixed one (figure $8 \mathrm{~b}$ ) and changes with system pressure (figure $8 \mathrm{c}$ ). The same characteristic is seen for increasing load moment at $0.03 \mathrm{~s}$. The above results indicate that with change in the bulk modulus, peak pressure as well as fluctuation of the fluid pressure increase in closed loop control applications. This increases the settling time of the system's responses. This may cause to failure of the stability of the system. Therefore, it is necessary to revise the control parameters or apply a more robust controller in terms of variable bulk modulus.

Fuzzy and PID control responses of HST system under the variable bulk modulus are depicted in figure 9. Simulation results of fuzzy controller show good performance compared with PID controller in tracking referenced velocity (figure 9a). In addition, fuzzy controller rejects the effect of loading on pressure dynamics (figure 9b). Figure 9c indicates that fuzzy controller minimizes the fluctuations of bulk modulus value. This is the reason why the fuzzy response is more robust. Figure $9 \mathrm{~d}$ shows that PID controller can work safely up to $200 \mathrm{~Hz}$, whereas a fuzzy controller is capable of enduring $400 \mathrm{~Hz}$. Therefore, a fuzzy controller 
(a)

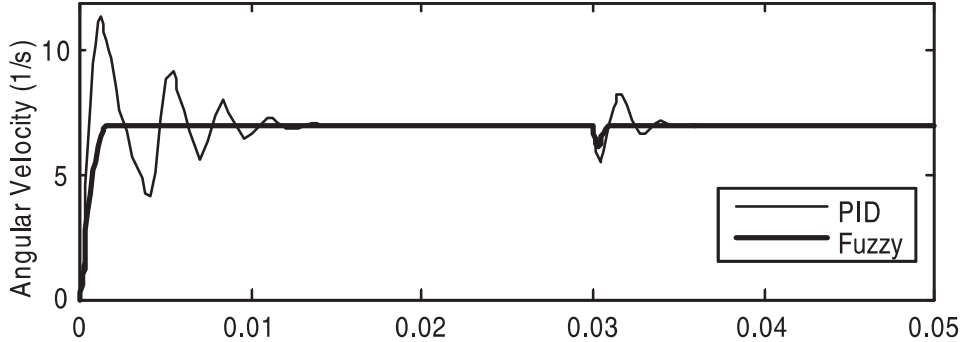

(b)

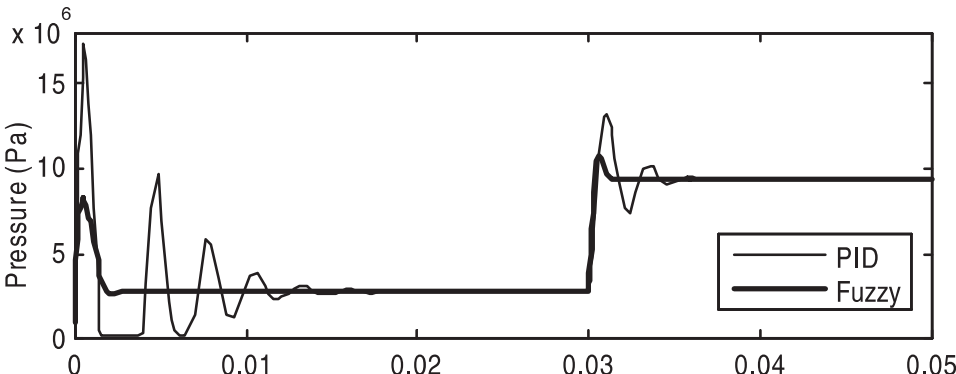

(c)

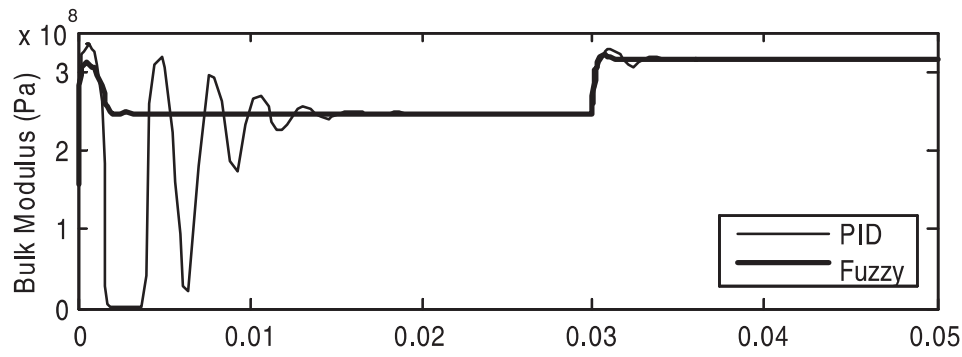

(d)

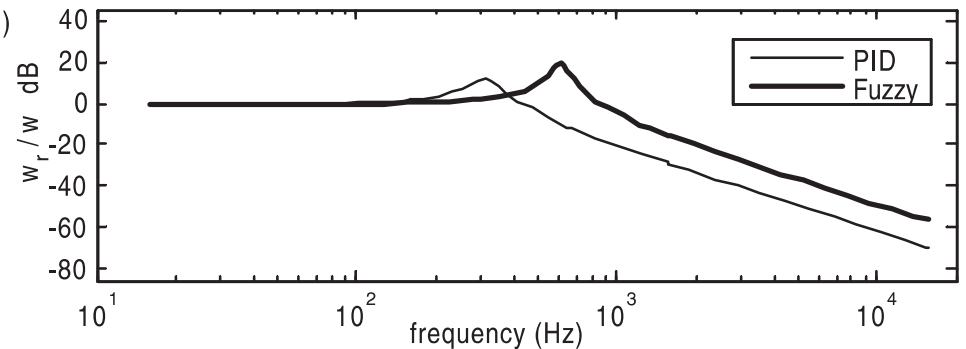

Figure 9. Fuzzy and PID control responses of HST system under variable bulk modulus. (a) Angular velocity, (b) system pressure, (c) variable bulk modulus, (d) velocity bode diagram.

increases the stability conditions of a hydraulic transmission system in presence of variable bulk nonlinearity.

\section{Conclusion}

The effects of bulk modulus nonlinearity on the performance of a hydrostatic transmission control system have been analysed through system modelling and simulation. This study has 
demonstrated that omitting the bulk modulus dynamics in hydrostatic transmission control systems may lead to major errors in system response and have implications on the safety of operation. Therefore, bulk modulus should be considered as a variable parameter to obtain a more realistic overall model and to determine more accurate control parameters in PID controller. Analysis including bulk modulus dynamics in an HST-control system model with this control design feature has not been described in the literature to date. Therefore, it may be useful for early design of an HST system used for PID control application. In addition, it is clearly seen that a fuzzy controller has the capability of eliminating the adverse effects of variable bulk modulus. This will also benefit the control design process in terms of developing a robust controller. For future research, model development will be expanded to include swashplate dynamics, valve dynamics and more complex flow and torque models of the pump and the motor. Furthermore, an adaptive control method will be applied for changeable velocity reference and load moment.

\section{List of symbols}

$B \quad$ viscous friction coefficient of motor and its shaft [Nms];

$B_{i} \quad$ centroid of the consequent fuzzy subset of $i$ th rule;

HST hydrostatic transmission;

$I_{m} \quad$ inertia of hydraulic motor shaft $\left[\mathrm{Nms}^{2}\right]$;

$k_{p} \quad$ pump coefficient $\left[\mathrm{m}^{3} /{ }^{\circ} \mathrm{s}\right]$;

$k_{m} \quad$ hydraulic motor coefficient $\left[\mathrm{m}^{3}\right]$;

$k_{m t} \quad$ motor torque coefficient $\left[\mathrm{m}^{3}\right]$;

$k_{v} \quad$ slope coefficient of static characteristic of relief valve $\left[\mathrm{m}^{5} / \mathrm{Ns}\right]$;

$M_{B} \quad$ moments resulted from friction force $[\mathrm{Nm}]$;

$M_{I} \quad$ moments resulted from load inertia $[\mathrm{Nm}]$;

$M_{m} \quad$ hydraulic motor torque [Nm];

$M_{o} \quad$ moments resulted from machine operation [Nm];

$P \quad$ system pressure [Pa];

$P_{v} \quad$ opening pressure value of valve $[\mathrm{Pa}] ;$

$Q_{c} \quad$ flow rate deal with compressibility $\left[\mathrm{m}^{3} / \mathrm{s}\right]$;

$Q_{m} \quad$ flow rate used in hydraulic motor $\left[\mathrm{m}^{3} / \mathrm{s}\right]$;

$Q_{p} \quad$ flow rate of pump $\left[\mathrm{m}^{3} / \mathrm{s}\right] ;$

$Q_{v} \quad$ passing flow rate through relief valve $\left[\mathrm{m}^{3} / \mathrm{s}\right]$;

$u v \quad$ output signal of fuzzy controller;

$V \quad$ fluid volume subjected to pressure effect $\left[\mathrm{m}^{3}\right]$;

$W_{i} \quad$ degree of firing of $i$ th fuzzy rule;

$\alpha \quad$ displacement angle of swashplate $\left[{ }^{\circ}\right]$;

$\beta \quad$ bulk modulus [Pa];

$\eta_{m m} \quad$ mechanical efficiency of hydraulic motor [-];

$\eta_{v p} \quad$ pump volumetric efficiency [-];

$\eta_{v m} \quad$ volumetric efficiency of motor $[-]$;

$\omega \quad$ angular velocity of motor $[1 / \mathrm{s}]$;

$\Delta P_{m} \quad$ pressure drop in hydraulic motor [Pa]. 


\section{References}

Dasgupta K 2000 Analysis of a hydrostatic transmission system using low speed high torque motor. Mech. Mach. Theory 35: 1481-1499

Dasgupta K, Chattapadhyay A, Mondal S K 2005 Selection of fire-resistant hydraulic fluids through system modelling and simulation. Simul. Model. Pract. Theory 13: 1-20

Eryilmaz B, Wilson B H 2001 Improved tracking control of hydraulic systems. Trans. ASME: J. Dyn. Syst. Meas. Control 123: 457-462

Huhtala K 1996 Modelling of hydrostatic transmission - steady state, linear and nonlinear models. Acta Polytech. Sci. Me. 123:

Jedrzykiewicz Z, Pluta J, Stojek J 1997 Research on the properties of a hydrostatic transmission for different efficiency models of its elements. Acta Montanistica Slovaca 2: 373-380

Jedrzykiewicz Z, Pluta J, Stojek J 1998 Application of the Matlab-Simulink package in the simulation tests on hydrostatic systems. Acta Montanistica Slovaca Rocnik 3: 29-36

Kugi A, Schlacher K, Aitzetmüller H, Hirmann G 2000 Modelling and simulation of a hydrostatic transmission with variable-displacement pump. Math. Comput. Simul. 53: 409-414

Lee C B, Wu H W 1996 Self-tuning adaptive speed control for hydrostatic transmission systems. Int. J. Comput. Appl. Technol. 9: 18-33

Lennevi J, Palmberg J O 1995 Application and implementation of LQ design method for the velocity control of hydrostatic transmissions. Proc. Inst. Mech. Eng., J. Syst. Control Eng. 209: 255-268

Manring N D 1997 The effective fluid bulk modulus within a hydrostatic transmission. Trans. ASME: J. Dyn. Syst. Meas. Control 119: 462-466

Manring N D, Luecke G R 1998 Modelling and designing a hydrostatic transmission with a fixeddisplacement motor. Trans. ASME: J. Dyn. Syst. Meas. Control 120: 45-49

McCloy D, Martin H R 1980 Control of fluid power, analysis and design (New York: John Wiley \& Sons)

Merrit H E 1967 Hydraulic control systems (New York: John Wiley \& Sons)

Ogata K 1990 Modern control engineering (Englewood Chiffs, NJ: Prentice-Hall)

Piotrowska A 2003 The control of the rotational speed of hydraulic engine in hydrostatic transmission by use of the module DSP. 28th ASR Seminar, Instruments and Control (Ostrava: VS̆B-TU) pp. 291297

Prasetiawan E A 2001 Modelling, simulation and control of an earthmoving vehicle powertrain simulator. M Sc thesis, Mechanical Engineering in Graduate College, University of Illinois, Urbana, Il

Re L, Goransson A, Astolfi A 1996 Enhancing hydrostatic gear efficiency through nonlinear optimal control strategies. Trans. ASME: J. Dyn. Syst. Meas. Control 118: 727-732

Tan H Z, Sepehri N 2002 Parametric fault diagnosis for electrohydraulic cylinder drive units. IEEE Trans. Ind. Electron. 49: 96-106

Tanaka K 1996 Introduction to fuzzy logic for engineering application (Berlin Springer)

Tikkanen S, Huhtala K, Vilenius M 1995 Fuzzy controllers in hydrostatic transmission. IEE Colloquium on Innovative Actuators for Mechatronic Systems (London: Inst. Elec. Eng.) 15/1-15/3

Watton J 1989 Fluid power systems: Modelling, simulation, analog and microcomputer control (Englewood Chiffs, NJ: Prentice-Hall)

Wu K, Zhang Q, Hansen 2004 Modelling and identification of a hydrostatic transmission hardwarein-the-loop simulator. Int. J. Vehicle Des. 34: 63-75

Yu J, Chen Z, Lu Y 1994 The variation of oil effective bulk modulus with pressure in hydraulic systems. Trans. ASME: J. Dyn. Syst. Meas. Control 116: 146-150

Zadeh L 1965 Fuzzy sets. Inf. Control 8: 338-353 